\title{
MODELO SIMPLIFICADO DE UNA INTERFAZ DE CONEXIÓN A LA RED BASADA EN UN CONVERTIDOR ELECTRÓNICO DE POTENCIA PARA ESTUDIOS DE RED EN RÉGIMEN DINÁMICO
}

\author{
Simplified Model of A GRID-ConneCtion \\ INTERFACE BAsed ON POWER EleCtroniC \\ Converter for Grid Studies in Dynamic Regime
}

\author{
Danny Ochoa ${ }^{1, *}$ (i)
}

Recibido: 02-02-2021, Recibido tras revisión: 09-04-2021, Aceptado: 22-04-2021, Publicado: 01-07-2021

\section{Resumen}

El cambio de paradigma experimentado por los sistemas eléctricos a nivel mundial ha propiciado una participación masiva de nuevos agentes energéticos: generación, almacenamiento y consumo. En la mayoría de los casos, estos agentes están dotados de convertidores electrónicos de potencia (CEP) para verter su energía a la red. Esta realidad ha impulsado el desarrollo de modelos analíticos muy sofisticados y detallados de CEP para estudiar el impacto de su interacción con la red en un entorno de simulación. No obstante, cuando se trata de estudiar redes de gran dimensión o con sus componentes desagregados, la enorme carga computacional requerida para simular un modelo detallado podría suponer una limitante para la realización de tales estudios. En este artículo se propone el diseño de un modelo simplificado de una interfaz de conexión a la red basada en CEP, útil para estudios de red mediante MATLAB/Simulink®. El modelo está concebido para representar, con una precisión numérica razonable, el comportamiento dinámico que tendrían ciertas variables eléctricas de interés de un modelo detallado y, al mismo tiempo, para conseguir una reducción significativa del tiempo de cómputo. Un análisis comparativo de los resultados numéricos, las dinámicas generadas y el tiempo de convergencia de los dos modelos permite validar la propuesta. Estos hitos conseguidos permiten cumplimentar los objetivos planteados en esta investigación.

Palabras clave: convertidor electrónico de potencia, conversión de energía, modulación por ancho de pulso, sistemas eléctricos de potencia

\section{Abstract}

The paradigm change experienced by worldwide power systems has led to a massive participation of new energy agents: generation, storage, and consumption. In most cases, these agents are equipped with power electronic converters (PEC) to incorporate their energy to the grid. This reality has motivated the development of highly sophisticated and detailed PEC analytical models that accurately represent their dynamics and enable to study their impact on the grid in a simulation environment. However, when it comes to studying large-scale power systems or with all their components disaggregated, the huge computational burden required to simulate a detailed model could make these studies unfeasible. This paper proposes the design of a simplified model of a grid-connection interface based on PEC for power system analysis using MATLAB/Simulink®. The model is designed to represent, with reasonable numerical accuracy, the dynamic behavior of certain electrical variables of interest that would produce a detailed model and, at the same time, to achieve a noticeable reduction in the computation time. A comparative analysis of the numerical results, the dynamics generated, and the convergence time achieved by the two models enable to validate the proposal. These milestones make it possible to fulfill the objectives of this research.

Keywords: Energy Conversion, Power Electronic Converter, Power Systems, Pulse Width Modulation

\footnotetext{
$\overline{1, *}$ Departamento de Ingeniería Eléctrica, Electrónica y Telecomunicaciones, Universidad de Cuenca, Ecuador. Autor para correspondencia danny.ochoac@ucuenca.edu.ec.
}

Forma sugerida de citación: Ochoa, D. (2021). «Modelo simplificado de una interfaz de conexión a la red basada en un convertidor electrónico de potencia para estudios de red en régimen dinámico». InGENIUS. N. ${ }^{\circ} 26$, (julio-diciembre). pp. 87-98. DOI: https://doi.org/10.17163/ings.n26.2021.08. 


\section{Introducción}

En las últimas décadas, los sistemas eléctricos de potencia han venido experimentando un cambio de paradigma que los ha obligado a pasar de un modelo de generación predominantemente centralizado a un modelo de generación más distribuido e incluso a un modelo de red eléctrica inteligente, junto con la inclusión de nuevos agentes energéticos de generación, de almacenamiento y de consumo $[1,2]$. En el marco de esta transición, cada vez se han ido integrado más generadores eléctricos renovables, vehículos eléctricos, sistemas de almacenamiento energético, entre otros, cuya interfaz de conexión a la red está basada en convertidores electrónicos de potencia (Figura 1).

Esto debido a que la electricidad generada/consumida por estos agentes es incompatible con la red en términos del tipo de energía (corriente continua o corriente alterna), la amplitud y la frecuencia de la tensión, etc. La necesidad de emplear convertidores electrónicos para verter la energía proveniente de generadores renovables (por ejemplo, eólicos o fotovoltaicos) a la red obedece a criterios de obtención de la máxima eficiencia en la conversión del recurso energético primario en energía eléctrica [3,4]. En el caso de las estaciones de carga de los vehículos eléctricos, la energía suministrada a las baterías es manejada por convertidores electrónicos de potencia con el objeto de garantizar la eficiencia del proceso y salvaguardar la vida útil de las mismas [5].

Situación similar se aplica para los sistemas de almacenamiento energético en baterías, en cuyo caso, el flujo energético puede ir de la batería a la red o al revés en función de la necesidad de la red en disponer o almacenar energía [6]. Los convertidores electrónicos de potencia poseen una topología que permite un flujo bidireccional de potencia activa y reactiva en función de las señales de consigna preestablecidas en los sistemas de control que gobiernan la lógica de disparo de los semiconductores que los conforman. Por tanto, desde la perspectiva de la red, estas interfaces se comportan como fuentes controladas de corriente [7].

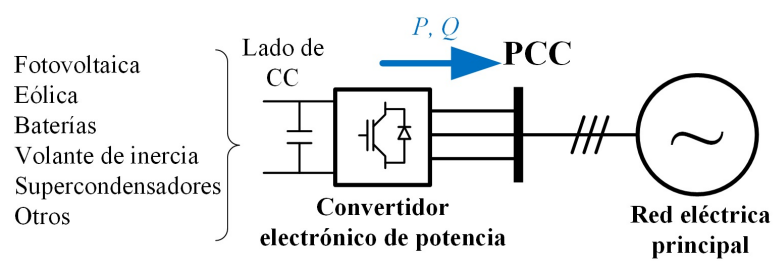

Figura 1. Convertidor electrónico de potencia conectado a la red

En la operación de un sistema eléctrico de potencia en régimen dinámico, una participación reducida de generadores renovables con interfaz de conexión a la red basada en convertidores electrónicos de potencia no supone un problema severo de estabilidad (de frecuencia y tensión), pues, esta labor es llevada a cabo de forma exitosa por la generación síncrona convencional. Sin embargo, a medida que esta integración se masifica (en sustitución de la generación síncrona), la red empieza a sufrir una disminución de sus características inerciales y, en consecuencia, la generación eléctrica convencional operativa puede resultar insuficiente para garantizar la estabilidad del sistema en caso de suscitarse alguna falla o contingencia [8].

Esta situación es de enorme preocupación en sistemas eléctricos débiles y aislados, en los cuales la cuota de participación de la generación renovable en el mix energético es equiparable o supera a la generación convencional, tal como lo muestran estudios publicados en [9-11]. No obstante, muchas de las soluciones que se proponen en la literatura para mitigar los inconvenientes antes expuestos están basados justamente en la utilización de más convertidores electrónicos, normalmente, asociados a sistemas de almacenamiento energético (baterías, volantes de inercia, supercondensadores, entre otros).

Con esta exposición inicial se pretende mostrar el protagonismo que han cobrado los convertidores electrónicos de potencia en la operación de un sistema eléctrico. el cual ha motivado el desarrollo de modelos muy detallados y completos concebidos para emular su comportamiento dinámico e interacción con la red y para ser utilizado en estudios basados en simulación por computadora $[12,13]$. No obstante, cuando el objeto de un estudio es el análisis dinámico de redes de gran dimensión o de sistemas eléctricos con todos sus componentes desagregados, la enorme carga computacional requerida para simular modelos detallados de convertidores dentro de un rango temporal comprendido desde los pocos segundos hasta un par de minutos (tiempo en el que se manifiestan los fenómenos físicos relacionados con la estabilidad de frecuencia/tensión en la red) podría limitar, en gran medida, la realización de este tipo de estudios.

Esta situación ha motivado la búsqueda de alternativas metodológicas que permitan modelar a los convertidores electrónicos de potencia de una forma menos compleja sin que esto perjudique su exactitud numérica respecto a los resultados que brindaría un modelo detallado tradicional. Por ejemplo, recientes trabajos publicados en [14-17] presentan técnicas efectivas para conseguir una simplificación significativa de la lógica de control de los convertidores electrónicos de potencia, todas estas basadas en el control predictivo de estados finitos basado en el modelo FCS-MPC, (por sus siglas en inglés).

Los resultados reportados en estas contribuciones muestran que el control de los convertidores mediante FCS-MPC permiten acortar el tiempo de ejecución de los lazos de control en comparación con aquel asociado a la implementación de controladores lineales clási- 
cos sin que exista penalización en su rendimiento. Sin embargo, este beneficio trae consigo la necesidad de realizar un mayor número de cálculos para cumplir con los objetivos de control. Para apalear esta situación, en estos artículos se proponen diferentes diseños de convertidores matriciales orientados a reducir el número de sectores necesarios para la descomposición vectorial de la tensión trifásica en el punto de conexión común, ya sea mediante una simplificación de la formulación empleada [15-17] o por medio de la utilización de tablas de búsqueda que agilicen el proceso de cálculo [14].

Un hecho que guardan en común estas contribuciones científicas es que las simplificaciones propuestas centran sus esfuerzos en optimizar la lógica de control del convertidor, pero manteniendo una representación detallada del inversor trifásico (puente de 6 o 9 transistores de potencia). En cambio, en [18] se plantea una idea de simplificación enfocada en el inversor trifásico, representado mediante un puente de seis fuentes controladas de corriente, moduladas mediante SPWM, cuyos réditos computacionales son latentes según lo evidencian los resultados reportados. Estos vislumbran también que las bondades obtenidas al simplificar la representación analítica del inversor trifásico son mermadas, en cierta medida, al utilizarse una técnica de modulación por ancho de pulso para el control de las fuentes de corriente controladas, la cual demanda de un importante esfuerzo computacional para su implementación. Es aquí en donde se ha identificado la brecha de investigación que ha dado lugar al planteamiento de la presente propuesta.

Con el objeto de conseguir un grado mayor de simplificación, al tiempo de mantener un compromiso entre la simplicidad y la exactitud, se propone el desarrollo de un modelo simplificado de una interfaz de conexión a la red basada en convertidores electrónicos de potencia para ser utilizado en estudios de red en régimen dinámico en el entorno de simulación MATLAB/Simulink®. El inversor trifásico es representado por tres fuentes de corriente controladas que son gobernadas por una pareja de controladores lineales en coordenadas $d-q$ diseñada para los efectos de esta investigación. Para ello, se toma como referencia la base teórica del principio de operación del modelo detallado de un convertidor electrónico de potencia completo y su implementación en el programa de simulación.

\section{Materiales y métodos}

La investigación desarrollada en este trabajo es del tipo experimental, por cuanto los experimentos, conducidos en un entorno de simulación por computadora, se realizan bajo condiciones controladas [19].

En primera instancia, se presenta la base teórica y la implementación de un modelo detallado de un convertidor electrónico de potencia (CEP). Luego se plantea el desarrollo del modelo simplificado concebido para emular el comportamiento dinámico que tendría el modelo detallado, desde el punto de vista de la red, dentro del horizonte temporal de interés delimitado en esta investigación. Al mismo tiempo, se busca conseguir una reducción significativa del esfuerzo computacional requerido para representar la dinámica de determinadas variables eléctricas manejadas por el CEP en la simulación. Estos son los objetivos principales de la investigación.

\subsection{Representación detallada de un conver- tidor electrónico de potencia}

La Figura 2 muestra la configuración típica de un CEP conectado a la red. En la ilustración, es posible distinguir dos subsistemas: el inversor trifásico y su controlador de potencia PQ. El inversor, constituido por un puente de semiconductores de potencia (SCR, MOSFET, IGBT, u otros, según la aplicación), tiene por función convertir la energía proveniente de un sistema de corriente continua (CC) en energía en corriente alterna $(\mathrm{CA})$ para su posterior vertido a la red trifásica, o viceversa. Según qué interruptores estén activados en cada instante de tiempo, el puente irá construyendo una señal de tensión trifásica en sus bornes cuya frecuencia fundamental será heredada de la tensión impuesta por la red en el punto de conexión común (PCC, por sus siglas en inglés) y, cuya amplitud y fase estarán definidas según qué corriente es necesaria inyectar para conseguir un valor predeterminado de potencia activa y reactiva en el PCC por parte del convertidor.

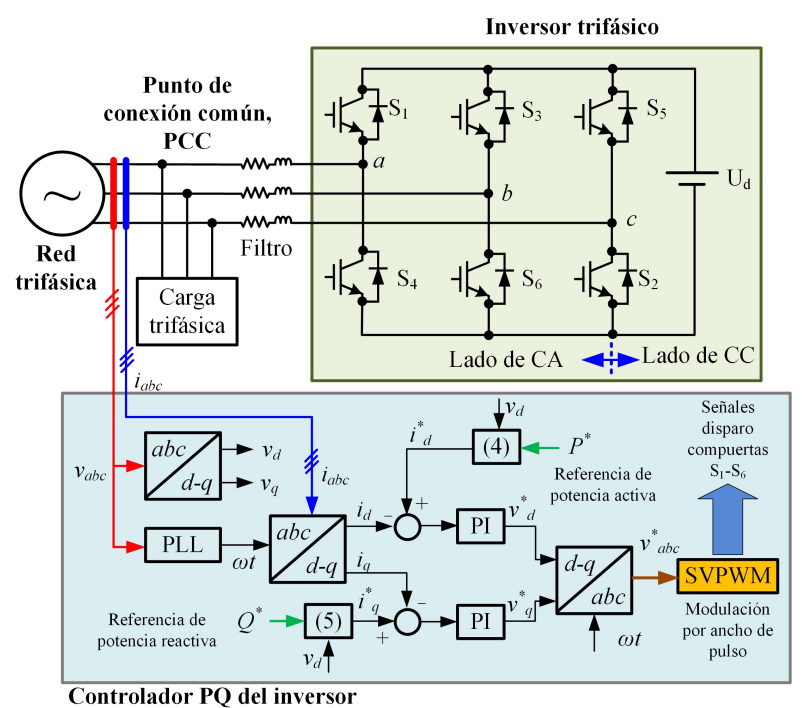

Figura 2. Configuración típica de un CEP trifásico conectado a la red

La secuencia de disparos aplicada a cada uno de los seis semiconductores del inversor se consigue mediante técnicas de modulación por ancho de pulso, cuyo 
principio se expone en la sección 2.1.1, mientras que, las labores de control de la potencia activa y reactiva inyectada se llevan a cabo mediante un esquema de control PQ similar a aquel mostrado en el subsistema inferior de la Figura 2, cuyos criterios se explican con algo más de detalle en la sección 2.1.2. Además, es preciso indicar que, con el objeto de reducir el contenido armónico de la señal de corriente inyectada por el CEP en el PCC, esta topología suele estar acompañada de un filtro pasivo trifásico de primer orden como se aprecia en la figura.

\subsubsection{Modulación de ancho de pulso mediante vectores espaciales (SVPWM)}

Para la generación de las señales de disparo de compuerta de los transistores que componen la topología del CEP, hoy en día se encuentran disponibles muchas técnicas con una gran aplicabilidad y alto grado de madurez, tales como: modulación por ancho de pulso (PWM, por sus siglas en inglés), PWM con eliminación selectiva de armónicos (SE-PWM), PWM con portadora sinusoidal (SPWM) y PWM mediante vectores espaciales (SVPWM) [20-22]. Con los recientes avances en la industria de los semiconductores, microprocesadores y en el procesamiento digital de señales, las técnicas de modulación por ancho de pulso mediante vectores espaciales se utilizan ampliamente en los CEP para aplicaciones de generación eléctrica debido a su mayor flexibilidad en el control, menor contenido armónico y mejor desempeño dinámico [22,23].

De forma general, la SVPWM es una técnica de modulación digital en la que un vector de referencia muestreado se sintetiza mediante un número apropiado de vectores de estado conmutados en determinados instantes de tiempo. Los vectores, tanto de referencia como los de estado conmutados, se representan en un plano complejo mediante una transformación de un marco referencial trifásico abc a un marco referencial de coordenadas $\alpha-\beta$. Para brindar al lector una primera aproximación al método, considérese el inversor trifásico mostrado en la Figura 2. Esta topología ofrece ocho estados de conmutación, los cuales, están arreglados de acuerdo con la secuencia a, b, c. Partiendo de la premisa de que en cada una de las tres ramas del puente de transistores puede estar activo (o en conducción) solamente uno de ellos, se procede a definir el estado de conmutación 1 cuando el transistor superior de la rama está activado y 0 cuando lo hace el transistor inferior de la misma.

Si se define a la tensión en el lado de CC del inversor como $U_{d}$, el inversor es capaz de ofrecer en sus terminales los valores de tensión (de fase $\boldsymbol{V}_{\boldsymbol{A}}, \boldsymbol{V}_{\boldsymbol{B}} \mathrm{y}$ $\boldsymbol{V}_{\boldsymbol{C}}$, y de línea $\boldsymbol{V}_{\boldsymbol{A} \boldsymbol{B}}, \boldsymbol{V}_{\boldsymbol{B} \boldsymbol{C}}, \boldsymbol{V}_{\boldsymbol{C} \boldsymbol{A}}$ ) que se indican en la Tabla 1. Ahora bien, el orden de selección de cada una de las ocho combinaciones de conmutación y el tiempo en el que estas deben mantenerse activas es lo que define la técnica SVPWM. La Figura 3 proporciona una idea gráfica de la mecánica de construcción del vector de tensión en los bornes del inversor, $\boldsymbol{V}_{\boldsymbol{n}}$, sobre el plano complejo estacionario $\alpha-\beta$. Este vector es descompuesto en ocho estados de conmutación: $\boldsymbol{V}_{\mathbf{0}}$ a $\boldsymbol{V}_{\mathbf{7}}$, de los cuales, $\boldsymbol{V}_{\mathbf{1}}-\boldsymbol{V}_{\mathbf{6}}$ son vectores activos que forman un hexágono regular (seis sectores) y, $\boldsymbol{V}_{\mathbf{0}}$ y $\boldsymbol{V}_{\mathbf{7}}$ son vectores nulos que reposan en el centro del hexágono. Una descripción detallada del marco teórico de esta técnica de modulación, el cálculo de los tiempos de conmutación para cada uno de los sectores y los criterios de implementación, pueden consultarse en [12,13,24].

Tabla 1. Características de las arquitecturas utilizadas

\begin{tabular}{ccccccccc}
\hline \multicolumn{1}{c}{ Estado } & \multicolumn{7}{c}{ Tensiones en bornes del inversor } \\
\hline$a$ & $b$ & $c$ & $\boldsymbol{V}_{\boldsymbol{A}}$ & $\boldsymbol{V}_{\boldsymbol{B}}$ & $\boldsymbol{V}_{\boldsymbol{C}}$ & $\boldsymbol{V}_{\boldsymbol{A} \boldsymbol{B}}$ & $\boldsymbol{V}_{\boldsymbol{B} \boldsymbol{C}}$ & $\boldsymbol{V}_{\boldsymbol{C} \boldsymbol{A}}$ \\
\hline 0 & 0 & 0 & 0 & 0 & 0 & 0 & 0 & 0 \\
0 & 0 & 1 & $-\mathrm{U}_{\mathrm{d}} / 3$ & $-\mathrm{U}_{\mathrm{d}} / 3$ & $2 \mathrm{U}_{\mathrm{d}} / 3$ & 0 & $-\mathrm{U}_{\mathrm{d}}$ & $\mathrm{U}_{\mathrm{d}}$ \\
0 & 1 & 0 & $-\mathrm{U}_{\mathrm{d}} / 3$ & $2 \mathrm{U}_{\mathrm{d}} / 3$ & $-\mathrm{U}_{\mathrm{d}} / 3$ & $-\mathrm{U}_{\mathrm{d}}$ & $\mathrm{U}_{\mathrm{d}}$ & 0 \\
0 & 1 & 1 & $-2 \mathrm{U}_{\mathrm{d}} / 3$ & $\mathrm{U}_{\mathrm{d}} / 3$ & $\mathrm{U}_{\mathrm{d}} / 3$ & $-\mathrm{U}_{\mathrm{d}}$ & 0 & $\mathrm{U}_{\mathrm{d}}$ \\
1 & 0 & 0 & $2 \mathrm{U}_{\mathrm{d}} / 3$ & $-\mathrm{U}_{\mathrm{d}} / 3$ & $-\mathrm{U}_{\mathrm{d}} / 3$ & $\mathrm{U}_{\mathrm{d}}$ & 0 & $-\mathrm{U}_{\mathrm{d}}$ \\
1 & 0 & 1 & $\mathrm{U}_{\mathrm{d}} / 3$ & $-2 \mathrm{U}_{\mathrm{d}} / 3$ & $\mathrm{U}_{\mathrm{d}} / 3$ & $\mathrm{U}_{\mathrm{d}}$ & $-\mathrm{U}_{\mathrm{d}}$ & 0 \\
1 & 1 & 0 & $\mathrm{U}_{\mathrm{d}} / 3$ & $\mathrm{U}_{\mathrm{d}} / 3$ & $-2 \mathrm{U}_{\mathrm{d}} / 3$ & 0 & $\mathrm{U}_{\mathrm{d}}$ & $-\mathrm{U}_{\mathrm{d}}$ \\
1 & 1 & 1 & 0 & 0 & 0 & 0 & 0 & 0 \\
\hline
\end{tabular}

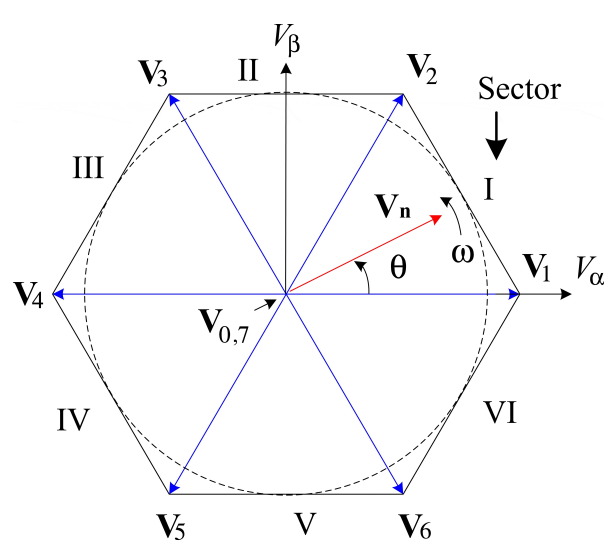

Figura 3. Modulación SVPWM

\subsubsection{Control de potencia $P Q$}

Para la mayoría de las aplicaciones industriales de los CEP, el control de potencia activa y reactiva se lleva a cabo mediante la transformación del vector trifásico de tensión y de corriente en vectores expresados en componentes de un sistema coordenado rotativo, en donde el vector de la variable se descompone en un vector de eje directo y en otro de cuadratura $(d-q)$ [25]. De la teoría de los sistemas eléctricos de potencia, el operador que permite transformar la representación de las variables eléctricas trifásicas $a b c$ a un marco referencial de coordenadas $d-q$ es la transformada de Park, tal y como se indica en (1). En esta ecuación, 
expresada para la corriente, $\boldsymbol{i}_{\boldsymbol{d}}, \boldsymbol{i}_{\boldsymbol{q}}$ e $\boldsymbol{i}_{\mathbf{0}}$ son las componentes en eje directo, en cuadratura y homopolar $(d q 0)$, respectivamente, de las corrientes trifásicas $\boldsymbol{i}_{\boldsymbol{a}}, \boldsymbol{i}_{\boldsymbol{b}}$ e $\boldsymbol{i}_{\boldsymbol{c}}$. $\omega$ es la pulsación angular de la red, que es la velocidad angular a la que gira el sistema coordenado $d-q$. En la Figura 4 se muestra un ejemplo de descomposición del vector corriente, $\boldsymbol{I}$, en las componentes: $\boldsymbol{I}_{\boldsymbol{d}}$ e $\boldsymbol{I}_{\boldsymbol{q}}$. Se puede aplicar un razonamiento similar para la tensión.

$$
\left[\begin{array}{l}
i_{d} \\
i_{q} \\
i_{0}
\end{array}\right]=\frac{2}{3} \underbrace{\left[\begin{array}{ccc}
\cos \omega t & \cos \left(\omega t-\frac{2 \pi}{3}\right) & \cos \left(\omega t+\frac{2 \pi}{3}\right) \\
-\operatorname{sen} \omega t & -\operatorname{sen}\left(\omega t-\frac{2 \pi}{3}\right) & -\operatorname{sen}\left(\omega t+\frac{2 \pi}{3}\right) \\
\frac{1}{2} & \frac{1}{2}
\end{array}\right]}_{\text {Transformada de park }}\left[\begin{array}{l}
i_{a} \\
i_{b} \\
i_{c}
\end{array}\right]
$$

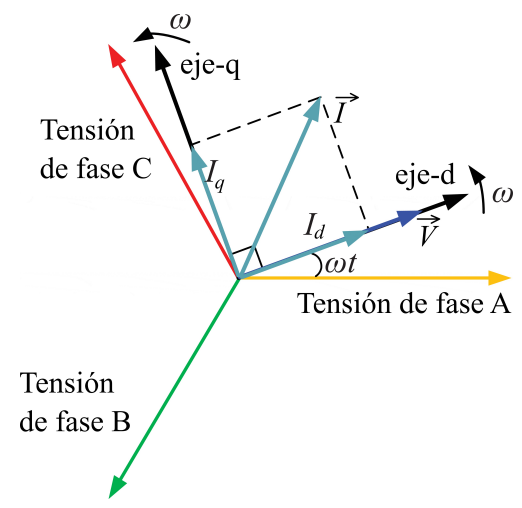

Figura 4. Sistema coordenado $d-q$

En la literatura se ha demostrado que el cálculo de las potencias instantáneas en un sistema trifásico puede realizarse por medio del cómputo de las variables instantáneas de tensión y corriente expresadas en coordenadas $d-q$, como sigue [26]:

$$
\begin{gathered}
P=\frac{3}{2}\left(v_{d} i_{d}+v_{q} i_{q}\right) \\
Q=\frac{3}{2}\left(-v_{d} i_{q}+v_{q} i_{d}\right)
\end{gathered}
$$

Donde:

$v_{d}$ e $i_{d}$ : tensión y corriente instantánea en eje directo.

$v_{q}$ e $i_{q}$ : tensión y corriente instantánea en eje en cuadratura.

Por conveniencia analítica, en este trabajo se forzará a que el eje directo del sistema coordenado d-q esté alineado con el vector tensión de fase, de tal forma que se consiga hacer la componente $v_{q}=0 \mathrm{y}$, además, que $v_{d}=|\mathbf{V}|=V$. Con este arreglo, las potencias instantáneas quedan definidas de la siguiente manera:

$$
P=\frac{3}{2} V i_{d}
$$

$$
Q=-\frac{3}{2} V i_{q}
$$

Este último par de expresiones vislumbra las ventajas de emplear las coordenadas $d-q$ para el control de la potencia inyectada por el CEP a la red, pues, la regulación de la potencia activa dependerá únicamente del manejo de la variable $i_{d}$, mientras que, el de la potencia reactiva, estará en función de $i_{q}$. Esto significa que el control de potencia $P$ pueden llevarse a cabo de forma desacoplada de las consignas aplicadas al control de potencia $Q$, siendo necesario para este propósito el diseño de un solo controlador por variable. En el recuadro inferior de la Figura 2 se muestra un ejemplo de implementación del controlador $\mathrm{PQ}$ para el inversor trifásico en estudio.

\subsection{Implementación del modelo detallado del convertidor electrónico de potencia en MATLAB/Simulink®}

En función de lo expuesto en los literales anteriores, en esta sección se presenta la implementación del modelo detallado de un convertidor electrónico de potencia en el entorno de simulación MATLAB/Simulink®. Se define como banco de pruebas la red ilustrada en la Figura 2, cuya implementación en el simulador se muestra en la Figura 5.

Debido a que el interés científico de este trabajo se centra en el estudio de la interacción del convertidor electrónico de potencia con la red, en el lado de CC del inversor se colocará una fuente de tensión ideal, la cual puede representar el recurso energético primario de distintos agentes como: generación fotovoltaica o eólica, volantes de inercia, supercondensadores, banco de baterías, etc.

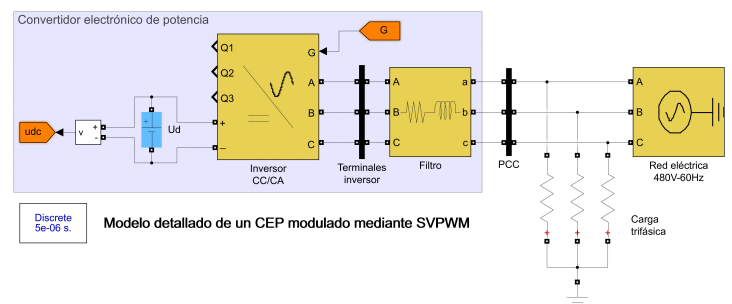

(a)

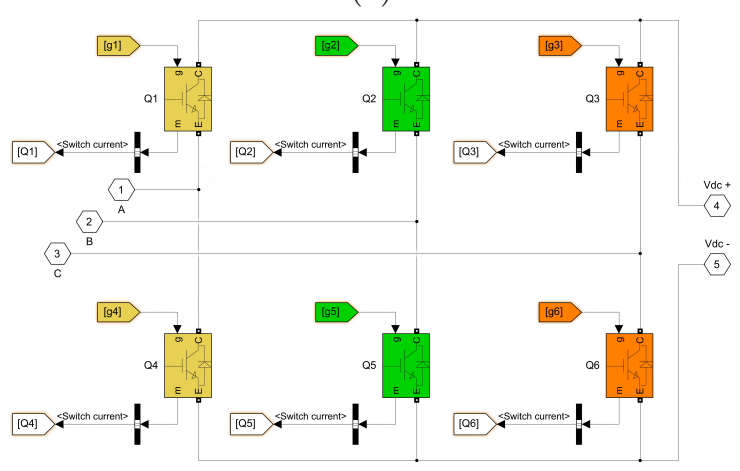

(b) 


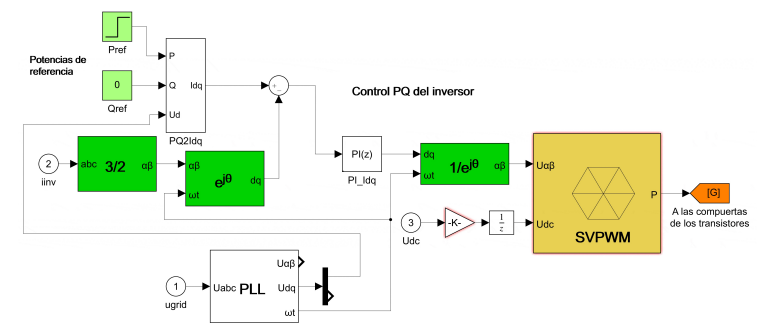

(c)

Figura 5. Implementación del CEP en MATLAB/Simulink® (modelo detallado): a) sistema eléctrico de pruebas; b) configuración del inversor y; c) controlador PQ y modulador SVPWM

Los valores asignados a los parámetros de los distintos elementos que constituyen el sistema de pruebas se proporcionan en el Apéndice. Si el lector precisa de mayores detalles para la implementación del convertidor en el simulador, se recomienda consultar [13].

Para evaluar el desempeño del modelo detallado del convertidor electrónico de potencia en el dominio del tiempo en el entorno de simulación, se establece un valor de potencia activa de referencia constante que inicie en $10 \mathrm{~kW}$ y, una vez que hayan transcurrido $0,4 \mathrm{~s}$ de simulación este cambie a $20 \mathrm{~kW}$. Además, se ha establecido una consigna de la potencia reactiva nula durante el horizonte temporal de simulación, a fin de verificar el control desacoplado $\mathrm{PQ}$ que ofrece la filosofía de control mediante coordenadas $d-q$. Las siguientes figuras muestran los resultados obtenidos en la simulación.

La Figura 6 ilustra la señal de tensión fase-neutro construida por el inversor trifásico mediante la aplicación de la modulación SVPWM y medida en los terminales del inversor (antes de la etapa de filtrado). Como se aprecia en las gráficas, esta tensión de fase oscila a la frecuencia fundamental de la red $(60 \mathrm{~Hz})$ y presenta una componente de alta frecuencia $(20 \mathrm{kHz})$ heredada de la portadora empleada para la modulación por ancho de pulso, la cual, será atenuada al pasar por el filtro R-L serie.

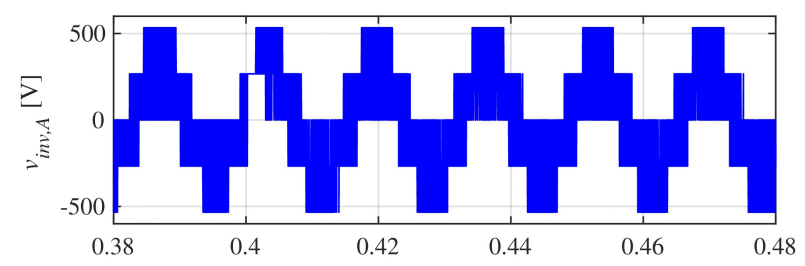

(a)

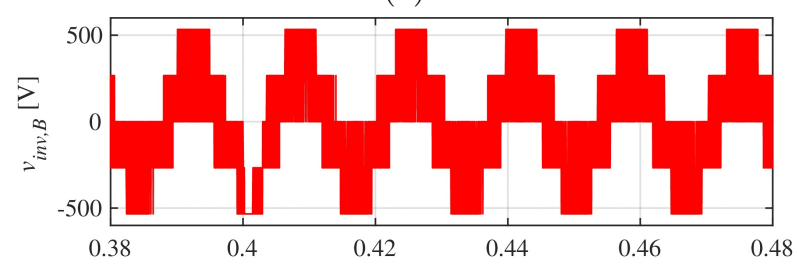

(b)

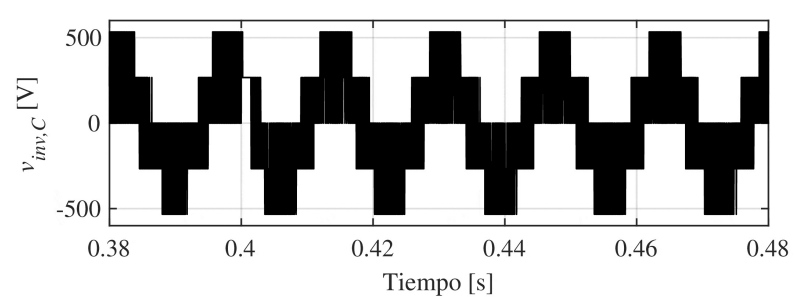

(c)

Figura 6. Tensión trifásica generada en bornes del inversor

La Figura 7a muestra la forma de onda de la corriente inyectada por el CEP en el PCC, la cual ha sido construida por el controlador $\mathrm{PQ}$ del inversor para el cumplimiento de las consignas de potencia activa y reactiva establecidas. La dinámica en el dominio del tiempo muestra el correcto desempeño del inversor y, además, la efectividad en las labores de filtrado (los valores de distorsión armónica total (THD) se aportan en la misma figura). En base a las mediciones de tensión y corriente en los bornes del CEP se han graficado, además, las dinámicas adoptadas por la potencia activa y reactiva inyectadas en el PCC (Figuras 7b y $7 \mathrm{c}$, respectivamente). Nótese en la Figura $7 \mathrm{~b}$ cómo el CEP sigue correctamente la consigna de potencia activa establecida en el sistema de control PQ.

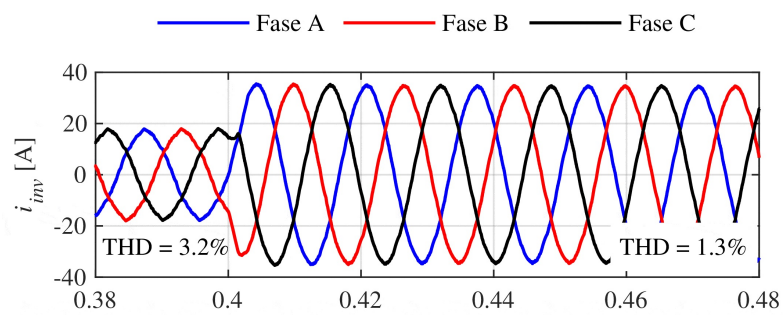

(a)

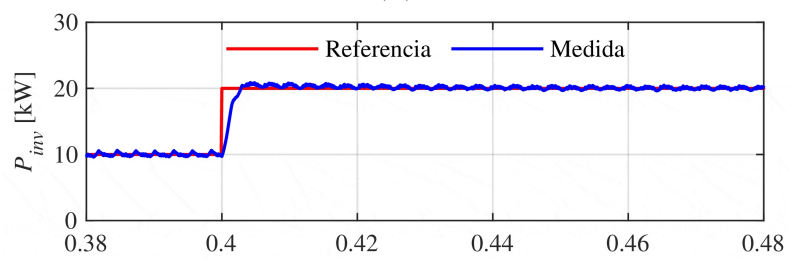

(b)

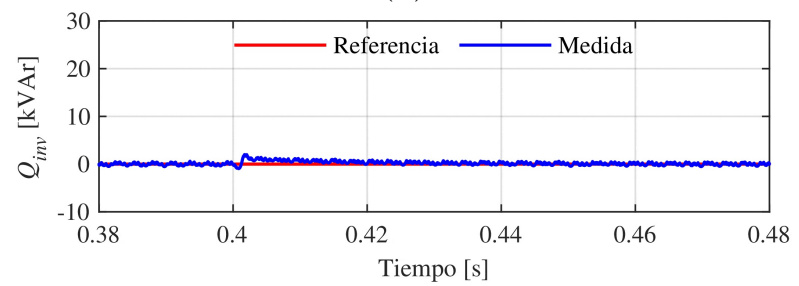

(c)

Figura 7. Variables eléctricas medidas a la salida del CEP: a) corriente; b) potencia activa y; c) potencia reactiva

Del mismo modo, en la Figura 7c se puede valorar la efectividad del control de la potencia reactiva, pues, 
esta variable es mantenida prácticamente en cero en todo el intervalo simulado. Además, esto último pone en manifiesto una de las bondades que ofrece el CEP en su integración a la red: el control desacoplado de potencias $P$ y $Q$.

Para el interés científico de este estudio, centrado en aquellos fenómenos que ocurren en un horizonte temporal en el orden de los milisegundos y de los pocos segundos en la dinámica operativa de un sistema eléctrico de potencia (como se justificó en la parte introductoria de este trabajo), las consignas tanto de potencia activa como de reactiva son alcanzadas de forma prácticamente instantáneas por el CEP. Esta apreciación será la premisa para el planteamiento de la modelación simplificada abordada en la siguiente subsección.

\subsection{Diseño del modelo simplificado}

En estudios de sistemas eléctricos de potencia en régimen dinámico, que tienen como objetivo evaluar el impacto de los agentes energéticos basados en interfaz de conexión a la red por medio de convertidores electrónicos de potencia, la utilización de un modelo detallado centrado en la arquitectura interior del CEP, como aquel presentado en la subsección anterior, puede demandar de un elevado esfuerzo computacional para la representación de sus variables internas y externas, el cual haría inviable muchos estudios en entornos de simulación en el caso de redes multimáquina y de gran dimensión. Este trabajo tiene como objetivo desarrollar un modelo simplificado que emule correctamente el comportamiento dinámico de un CEP visto desde la red en el punto de conexión común (PCC), medido en términos de sus variables eléctricas. Para ello, tanto el principio de operación, los criterios de control y los resultados de simulación descritos en la sección 2.1, servirán de punto de partida para el planteamiento de la propuesta.

\subsubsection{Criterios de diseño}

En primer lugar, se pide considerar el diagrama de la Figura 8a. Esta ilustración es una representación sintetizada del sistema eléctrico mostrado en la Figura 2. De acuerdo con el modo de operación del CEP, este inyectará en el PCC unos valores de potencia activa y reactiva, $P_{i n v}$ y $Q_{i n v}$, respectivamente, de acuerdo con sus valores de consigna preestablecidos. Debido a que la tensión trifásica en el PCC está impuesta por la red (en amplitud y frecuencia), el CEP tendrá que inyectar una terna de corrientes tales que permitan alcanzar las potencias de referencia $P_{i n v}^{*}$ y $Q_{i n v}^{*}$, como se ha visto en los resultados ilustrados en la Figura 7. Por tanto, desde el punto de vista de la red en el PCC, el CEP se comporta como una fuente de corriente trifásica controlada (Figura 7a) cuya amplitud y fase dependerá, como ya se ha mencionado, de los valores de consigna de potencia. De acuerdo con la teoría de las componentes $d-q$ (sección 2.1.2), las corrientes trifásicas inyectadas están relacionadas de forma directa con las variables $P_{i n v}$ y $Q_{i n v}$, dada su dependencia con las componentes de corriente id e iq, respectivamente (Ecuaciones 4 y 5 ). La Figura 8b ilustra el criterio de simplificación del modelo del CEP, al representarlo como una fuente de corriente controlada, cuyo esquema de control se desarrolla a continuación.

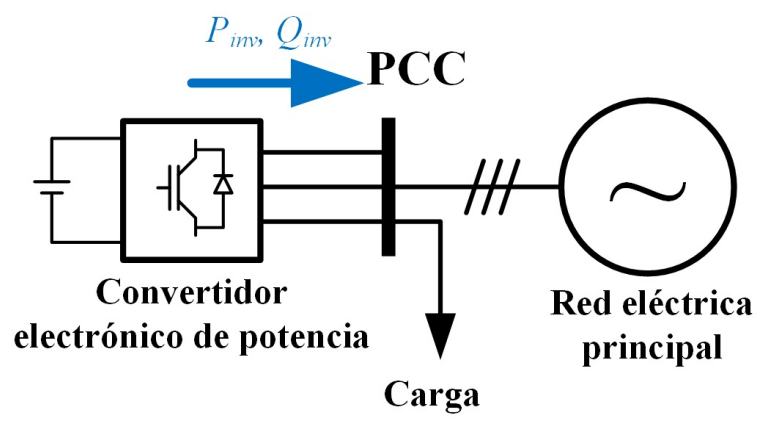

(a)

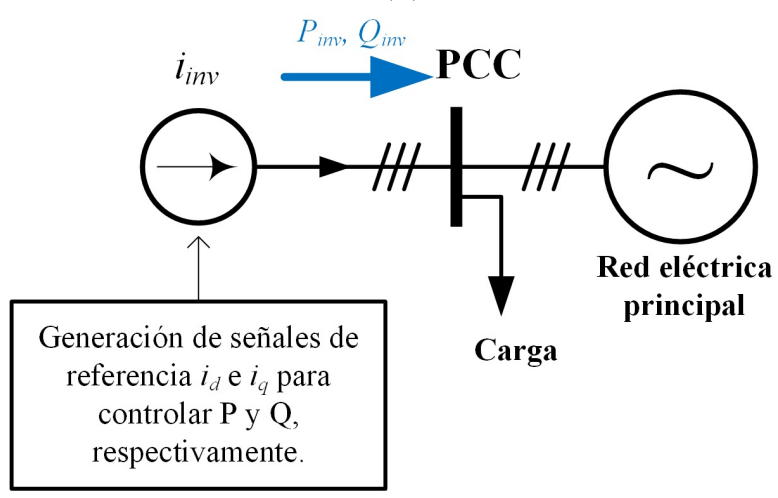

(b)

Figura 8. Criterio de simplificación del modelo del CEP: a) diagrama general; b) representación simplificada

La Figura 9 muestra la representación trifásica del diagrama de la Figura 8b, en la cual el CEP es modelado por medio de una fuente controlada de corriente. Esta fuente deberá inyectar unas corrientes trifásicas $i_{i n v, A}, i_{i n v, B}$ e $i_{i n v, C}$ cuya amplitud y fase serán definidas de acuerdo con el criterio de control implementado y las aplicaciones asignadas al convertidor.

Para la generación de las señales de consigna de corriente, en este trabajo se propone la utilización del esquema de control mostrado en la Figura 10. En este, las señales trifásicas aplicadas a la fuente controlada de corriente son generadas por medio de la aplicación de la teoría del sistema coordenado $d-q$, como se explica a continuación:

- Para el control de la potencia activa, $P_{i n v}^{*}$, se emplea un controlador en lazo cerrado PI, tal que 
genere en su salida una señal denominada $i_{d}^{*}$ que será la encargada de su regulación (Ecuación 4). Esta señal es aplicada a una función de retardo de primer orden, introducida para representar el tiempo que le toma al convertidor alcanzar en sus terminales el valor de la variable de control, $i_{d}$, desde que esta es especificada en su entrada, $i_{d}^{*}$.

- Se propone un esquema de control similar para la regulación de la potencia reactiva, $Q_{i n v}^{*}$, cuya regulación se efectúa de forma análoga a la del esquema descrito anteriormente, pero, en este caso, manipulando la variable $i_{q}$ (Ecuación 5).

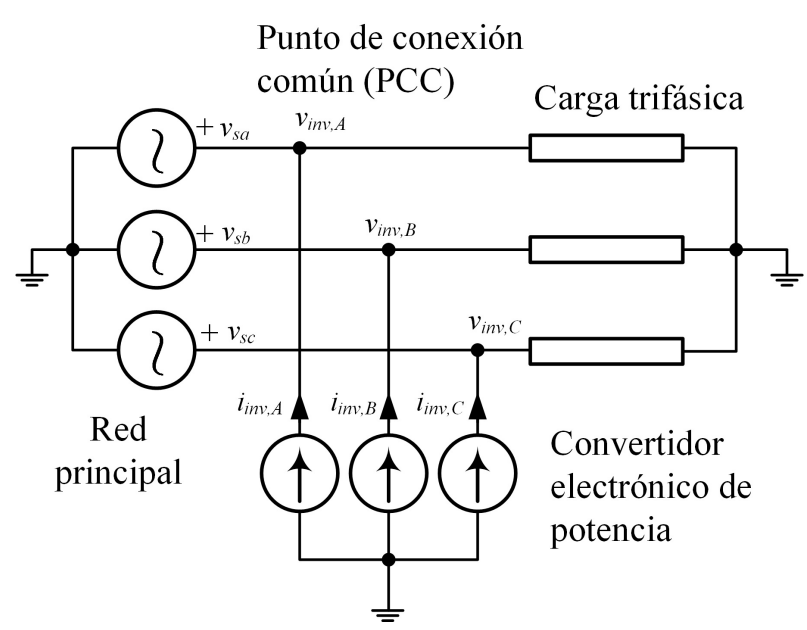

Figura 9. Propuesta de simplificación del CEP

Una vez generadas las señales $i_{d}$ e $i_{q}$, estas tienen que ser transformadas a un marco referencial trifásico $a b c$ antes de ser aplicadas a la fuente controlada que representa la dinámica del CEP. Para llevar a cabo esta transformación, la fase de la tensión en el PCC es medida en tiempo real por medio de un lazo cerrado de fase (PLL, por sus siglas en inglés) para luego proceder a evaluar la transformada de Park por medio de (1). Finalmente, para retroalimentar a los controladores PI de cada uno de los lazos de control, se miden las potencias instantáneas activa y reactiva inyectadas por las fuentes controladas de corriente en el PCC.

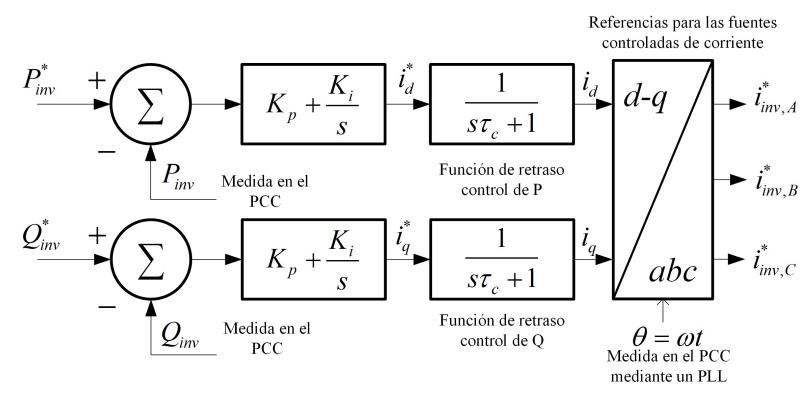

Figura 10. Propuesta de simplificación del controlador PQ

\subsubsection{Implementación de la propuesta en MATLAB/Simulink ${ }^{\circledR}$}

La Figura 11 muestra la implementación del modelo simplificado del CEP en el simulador sobre el mismo banco de pruebas empleado en la sección 2.1.3. Es de destacar la notoria simplificación en cuanto a la complejidad y número de elementos necesarios para representar la integración del CEP en la red, en comparación con el modelo de la Figura 5. Para facilitar la reproducción de los resultados de la simulación aquí expuestos, se pide al lector revisar los valores asignados a los distintos parámetros del modelo en el Apéndice.

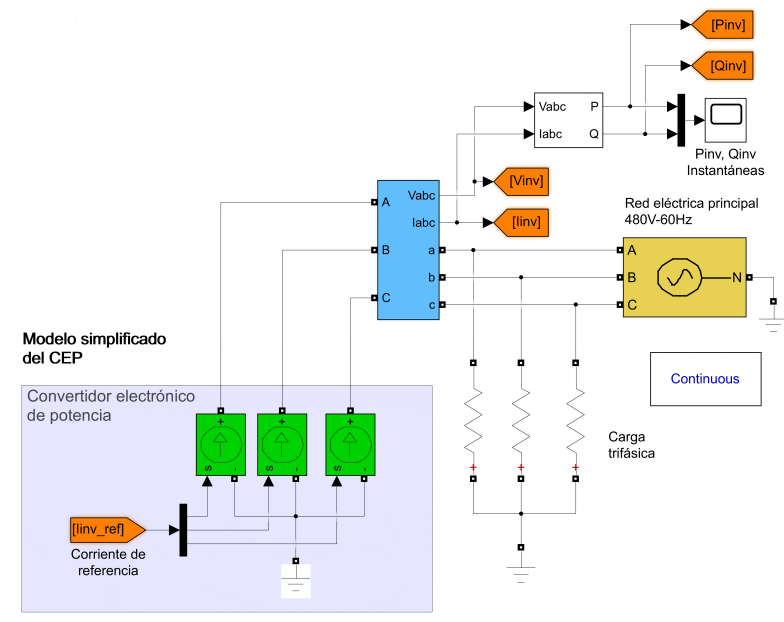

(a)

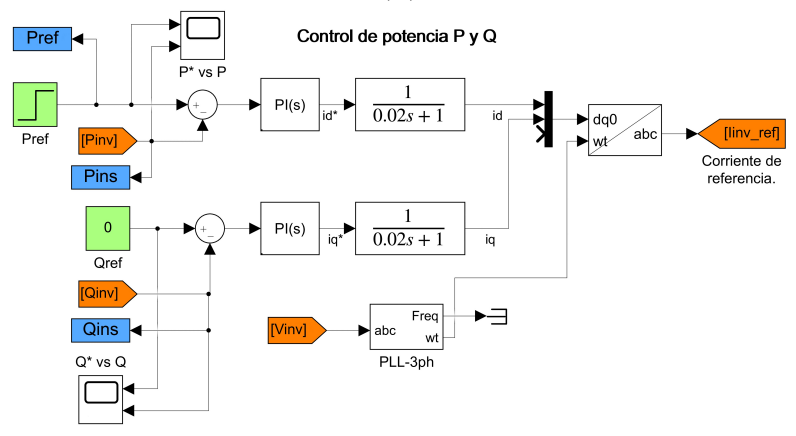

(b)

Figura 11. Esquema de implementación del modelo simplificado: a) sistema eléctrico de pruebas; b) controlador PQ

A continuación, se somete al modelo propuesto a operar bajo las mismas condiciones operativas definidas para simular el modelo detallado: señales de consigna de potencia activa y reactiva, horizonte de tiempo de la simulación, red eléctrica principal, carga alimentada, etc. Los resultados ilustrados en la Figura 12a revelan que la corriente trifásica inyectada por el CEP es generada correctamente y que su amplitud y forma es muy aproximada a aquella obtenida al simular el modelo detallado (Figura 7a). 
Las Figuras 12b y 12c muestran las potencias instantáneas activas y reactivas medidas en bornes del convertidor. En estas se observa que los respectivos valores de consigna son alcanzados exitosamente y de una forma más rápida que el modelo detallado. Debido a que el modelo simplificado propuesto se acerca más a las condiciones ideales de funcionamiento de un convertidor electrónico de potencia, las corrientes trifásicas carecen prácticamente de contenido armónico y, además, las potencias instantáneas alcanzan su valor objetivo de forma mucho más inmediata.

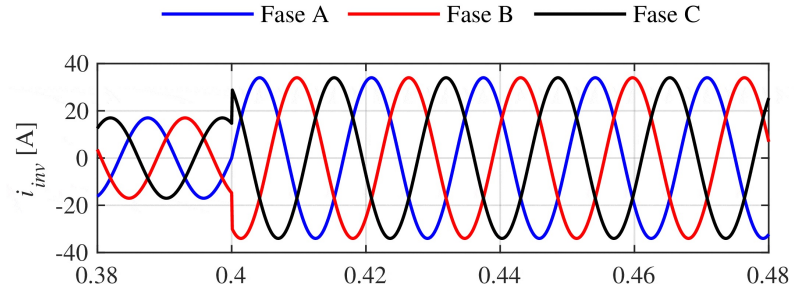

(a)

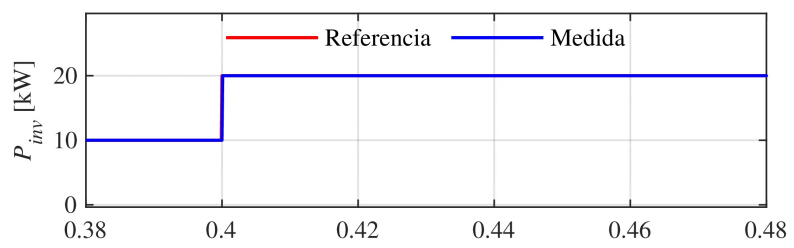

(b)

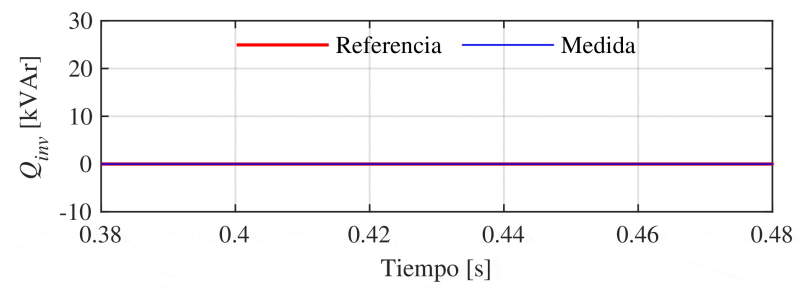

(c)

Figura 12. Variables eléctricas medidas a la salida del modelo simplificado del convertidor: a) corriente; b) potencia activa $\mathrm{y}$; c) potencia reactiva

\section{Resultados y discusión}

En esta sección se presenta un análisis comparativo del rendimiento del modelo simplificado propuesto (Figura 11) respecto al modelo detallado tomado como referencia (Figura 5). Para el efecto, a ambos modelos se aplican las señales de consigna de potencia mostradas en la Figura 13 y se los somete a las mismas condiciones operativas en el banco de pruebas diseñado en MATLAB/Simulink®.

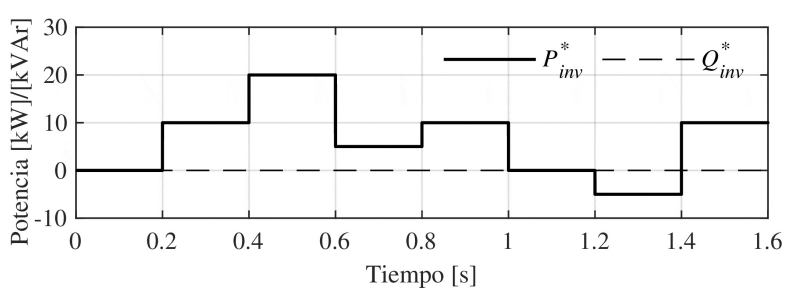

Figura 13. Perfil de las potencias de consigna aplicadas al convertidor

La Figura 14 muestra los resultados obtenidos en la simulación. En esta se observa el correcto desempeño de los dos modelos en el seguimiento de los valores de potencia de consigna asignados. Esta es una conclusión importante, pues estas variables son el resultado de cuantificar la tensión y la corriente trifásicas en los bornes del CEP y son aquellas que más interesan en el estudio dinámico de sistemas eléctricos de potencia. La corriente trifásica conseguida por el modelo propuesto tiene prácticamente la misma envolvente de aquella corriente generada por el modelo detallado. La similitud presentada por la dinámica de las variables de interés de los dos modelos, en el pequeño horizonte temporal graficado, permiten verificar la versatilidad del modelo propuesto y que la simplificación en la representación de los principales componentes de un CEP no penaliza de forma importante su exactitud numérica. Nótese que, la dinámica resultante corresponde al comportamiento ideal de un convertidor: transiciones de potencia mucho más inmediatas, ausencia de rizado en las potencias inyectadas y carencia de contenido armónico en la señal de corriente. Cuanto mayor sea el horizonte temporal contemplado en el estudio de red desarrollado con el modelo propuesto, menor será el impacto de estas limitaciones sobre los resultados numéricos generados.

Finalmente, en cuanto a los réditos computacionales obtenidos con la implementación de la propuesta, la simulación del modelo simplificado arroja sus resultados numéricos en un $4 \%$ del total del tiempo de cómputo que le toma al modelo detallado para converger. 

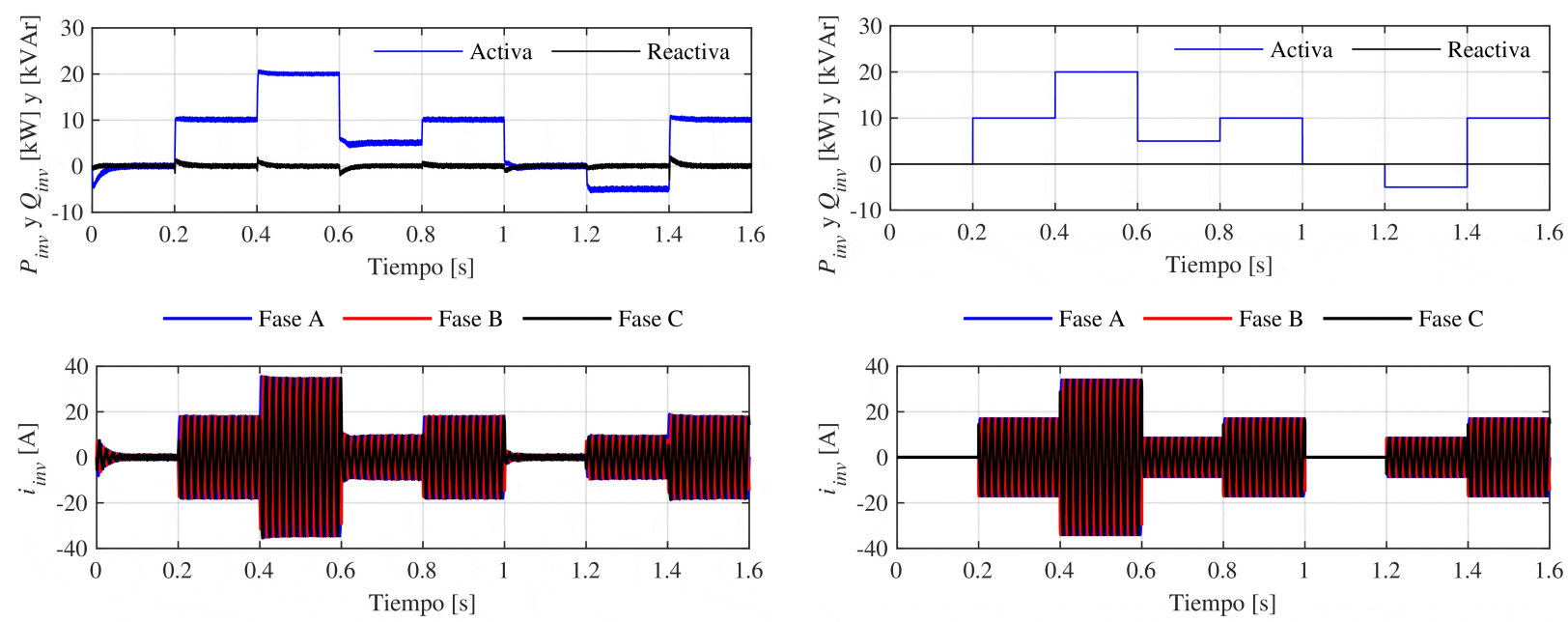

(a)

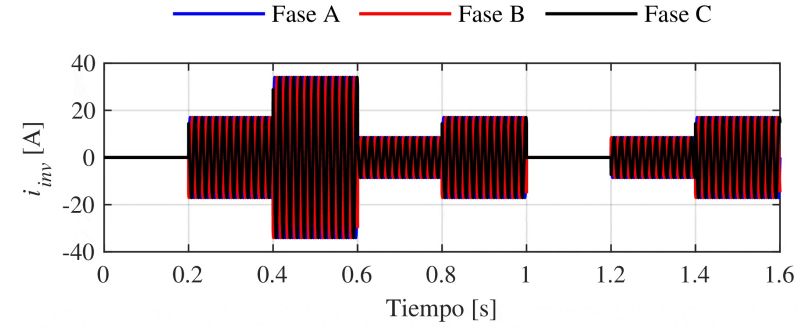

(b)

Figura 14. Resultados de la simulación: a) modelo detallado; b) modelo simplificado

\section{Conclusiones}

En este trabajo se ha desarrollado un modelo simplificado de una interfaz de conexión a la red basada en convertidor electrónico de potencia para estudios de red en régimen dinámico. Para el efecto, en primera instancia, se ha realizado una revisión exhaustiva del principio de operación de un convertidor electrónico de potencia y de su implementación en MATLAB/Simulink®. Luego, de acuerdo con la base teórica expuesta y al analizar los resultados preliminares de la simulación, se procedió a desarrollar un modelo simplificado del convertidor tal que permita emular un comportamiento dinámico similar al que se obtendría al simular un modelo detallado.

Los esfuerzos invertidos en las labores de simplificación y de diseño quedan justificados luego de realizar un análisis comparativo de las dinámicas generadas por los dos modelos al estar sometidos a las mismas condiciones operativas en el entorno de simulación. Los resultados numéricos y las dinámicas adoptadas por ciertas variables de interés son muy aproximados entre sí, consiguiéndose, además, una reducción del tiempo de cómputo numérico en el orden del $96 \%$. Estos hitos permiten cumplimentar los objetivos principales de este trabajo de investigación.

Es preciso indicar que el modelo propuesto arroja resultados numéricos que son más próximos al comportamiento ideal de un convertidor electrónico de potencia, por tanto, queda abierta la posibilidad de incorporar ciertas mejoras en investigaciones futuras, tales como:

- Agregar cierto contenido armónico a la señal de corriente trifásica inyectada en función de su amplitud.
- Realizar un proceso más exhaustivo de sintonización de las ganancias proporcional-integral del controlador PQ.

- Ejecutar técnicas de modelación, como por ejemplo, «hardware-in-the-loop», para conseguir una caracterización más realista de la función de retraso asignada a los lazos del controlador de potencia activa y reactiva, entre otras.

\section{Apéndice}

A. Parámetros red eléctrica:

Fuente trifásica: $V_{a b(r m s)}=480 \mathrm{~V}, \mathrm{f}=60 \mathrm{~Hz}$

Carga trifásica: $P_{L A}=P_{L B}=P_{L C}=1,00 \mathrm{~kW}$.

B. Parámetros modelo detallado:

Modulación SVPWM: $f_{\text {carrier }}=20 \mathrm{kHz}$

Controlador PI: $K_{P}=50$ y $K_{I}=2500$

Filtro R-L-serie: $R_{f}=0.1 \Omega$ y $L_{f}=12,7 \mathrm{mH}$

Tensión en CC: $U_{d}=800 \mathrm{~V}$

C. Parámetros modelo simplificado:

Controlador de P: $K_{p}=5$ y $K_{i}=50$

Controlador de Q: $K_{p}=-5$ y $K_{i}=-50$

Cte. tiempo función de retraso: $\tau_{C}=0,02 \mathrm{~s}$

D. Parámetros simulador

\begin{tabular}{ccc}
\hline & $\begin{array}{c}\text { Modelo } \\
\text { detallado }\end{array}$ & $\begin{array}{c}\text { Modelo } \\
\text { simplificado }\end{array}$ \\
\hline Solver & $\begin{array}{c}\text { ode3 } \\
\text { (Bogacki- } \\
\text { Shampine) }\end{array}$ & $\begin{array}{c}\text { ode23tb } \\
(\text { stiff } / \text { TR- } \\
\text { BDF2) }\end{array}$ \\
\hline $\begin{array}{c}\text { Tamaño de } \\
\text { paso cálculo } \\
\text { numérico }\end{array}$ & $5 \times 10^{-6} \mathrm{~s}($ fijo $)$ & $\begin{array}{c}0,5 \times 10^{-3} \mathrm{~s} \\
(\text { máx. variable) }\end{array}$ \\
\hline
\end{tabular}




\section{Referencias}

[1] I. Alotaibi, M. A. Abido, M. Khalid, and A. V. Savkin, "A comprehensive review of recent advances in smart grids: A sustainable future with renewable energy resources," Energies, vol. 13, no. 23, 2020. [Online]. Available: https://doi.org/10.3390/en13236269

[2] T. Sadamoto, A. Chakrabortty, T. Ishizaki, and J.-i. Imura, "Dynamic modeling, stability, and control of power systems with distributed energy resources: Handling faults using two control methods in tandem," IEEE Control Systems Magazine, vol. 39, no. 2, pp. 34-65, 2019. [Online]. Available: https://doi.org/10.1109/MCS.2018.2888680

[3] J. Baran and A. Jąderko, "An MPPT control of a PMSG-based WECS with disturbance compensation and wind speed estimation," Energies, vol. 13, no. 23, 2020. [Online]. Available: https://doi.org/10.3390/en13236344

[4] C. González-Castaño, L. L. Lorente-Leyva, J. Muñoz, C. Restrepo, and D. H. PeluffoOrdóñez, "An MPPT strategy based on a surfacebased polynomial fitting for solar photovoltaic systems using real-time hardware," Electron$i c s$, vol. 10, no. 2, 2021. [Online]. Available: https://doi.org/10.3390/electronics10020206

[5] O. Saadeh, A. Al Nawasrah, and Z. Dalala, "A bidirectional electrical vehicle charger and grid interface for grid voltage dip mitigation," Energies, vol. 13, no. 15, 2020. [Online]. Available: https://doi.org/10.3390/en13153784

[6] A. R. Choudhury, S. Pati, A. Choudhury, and K. B. Mohanty, "Control of voltage frequency of a hybrid microgrid using a FLC based bidirectional converter equipped with BESS," in 2018 Technologies for Smart-City Energy Security and Power (ICSESP), 2018, pp. 1-6. [Online]. Available: https://doi.org/10.1109/ICSESP.2018.8376666

[7] D. Li, F. Li, D. Rong, K. Zheng, D. Wang, and Q. Li, "An svpwm strategy for multifunction current source converter," in 2018 IEEE International Power Electronics and Application Conference and Exposition (PEAC), 2018, pp. 1-6. [Online]. Available: https://doi.org/10.1109/PEAC.2018.8590656

[8] A. Fernández-Guillamón, E. Gómez-Lázaro, E. Muljadi, and A. Molina-García, "Power systems with high renewable energy sources: A review of inertia and frequency control strategies over time," Renewable and Sustainable Energy Reviews, vol. 115, p. 109369, 2019. [Online]. Available: https://doi.org/10.1016/j.rser.2019.109369
[9] N. Bouzounierakis, Y. Katsigiannis, K. Fiorentzis, and E. Karapidakis, "Effect of hybrid power station installation in the operation of insular power systems," Inventions, vol. 4, no. 3, 2019. [Online]. Available: https://doi.org/10.3390/inventions4030038

[10] J. I. Sarasúa, G. Martínez-Lucas, C. A. Platero, and J. A. Sánchez-Fernández, "Dual frequency regulation in pumping mode in a wind-hydro isolated system," Energies, vol. 11, no. 11, 2018. [Online]. Available: https://doi.org/10.3390/en11112865

[11] D. Ochoa and S. Martínez, "Proposals for enhancing frequency control in weak and isolated power systems: Application to the wind-diesel power system of San Cristobal island-Ecuador," Energies, vol. 11, no. 4, 2018. [Online]. Available: https://doi.org/10.3390/en11040910

[12] A. Kocalmis and S. Sunter, "Simulation of a space vector pwm controller for a three-level voltage-fed inverter motor drive," in IECON 2006 - 32nd Annual Conference on IEEE Industrial Electronics, 2006, pp. 1915-1920. [Online]. Available: https://doi.org/10.1109/IECON.2006.347442

[13] S. Huang, D. C. Pham, K. Huang, and S. Cheng, "Space vector PWM techniques for current and voltage source converters: A short review," in 2012 15th International Conference on Electrical Machines and Systems (ICEMS), 2012, pp. 1-6. [Online]. Available: https://bit.ly/3fqdEdU

[14] M. Siami, D. A. Khaburi, M. Rivera, and J. Rodríguez, "A computationally efficient lookup table based FCS-MPC for PMSM drives fed by matrix converters," IEEE Transactions on Industrial Electronics, vol. 64, no. 10 , pp. $7645-7654,2017$. [Online]. Available: https://doi.org/10.1109/TIE.2017.2694392

[15] M. Siami, D. Arab Khaburi, and J. Rodríguez, "Simplified finite control set-model predictive control for matrix converter-fed PMSM drives," IEEE Transactions on Power Electronics, vol. 33, no. 3 , pp. 2438-2446, 2018. [Online]. Available: https://doi.org/10.1109/TPEL.2017.2696902

[16] T.-L. Nguyen, H.-N. Nguyen, T. D. Nguyen, and H.-H. Lee, "Simplified model predictive control for AC/DC matrix converters with fixed switching frequency," in 2019 10th International Conference on Power Electronics and ECCE Asia (ICPE 2019 - ECCE Asia), 2019, pp. 16. [Online]. Available: https://doi.org/10.23919/ ICPE2019-ECCEAsia42246.2019.8797210 
[17] J. Lei, S. Feng, P. Wheeler, B. Zhou, and J. Zhao, "Steady-state error suppression and simplified implementation of direct source current control for matrix converter with model predictive control," IEEE Transactions on Power Electronics, vol. 35, no. 3, pp. 3183-3194, 2020. [Online]. Available: https://doi.org/10.1109/TPEL.2019.2928874

[18] M. M. Bhesaniya and A. Shukla, "Computationally efficient method for simulating current source modular multilevel converter," in 2016 18th European Conference on Power Electronics and Applications (EPE'16 ECCE Europe), 2016, pp. 1-10. [Online]. Available: https://doi.org/10.1109/EPE.2016.7695490

[19] R. Hernández Sampieri, C. Fernández Collado, and P. Baptista Lucio, Metodología de la investigación. McGraw-Hill Education, 2014. [Online]. Available: https://bit.ly/3vqDKD4

[20] A. Moeed Amjad and Z. Salam, "A review of soft computing methods for harmonics elimination PWM for inverters in renewable energy conversion systems," Renewable and Sustainable Energy Reviews, vol. 33, pp. 141-153, 2014. [Online]. Available: https://doi.org/10.1016/j.rser.2014.01.080

[21] K. Latha Shenoy, C. G. Nayak, and R. P. Mandi, "MPPT enabled SPWM based bipolar VSI design in photovoltaic applications," $\mathrm{Ma}$ terials Today: Proceedings, vol. 5, no. 1, Part
1, pp. 1372-1378, 2018. [Online]. Available: https://doi.org/10.1016/j.matpr.2017.11.223

[22] A. Arif, Y. Bekakra, D. B. Attous, and H. Bakini, "Comparative study between indirect power control and sliding mode control with SVPWM for DFIG driven by wind turbine," in 2020 1st International Conference on Communications, Control Systems and Signal Processing (CCSSP), 2020, pp. 322-327. [Online]. Available: https: //doi.org/10.1109/CCSSP49278.2020.9151770

[23] R. Palanisamy, P. Aravindh, and K. Vijayakumar, "Experimental and numerical analysis on SVPWM based grid connected photovoltaic system," Materials Today: Proceedings, 2020. [Online]. Available: https://doi.org/10.1016/j.matpr.2020.08.382

[24] M. A. A. Badran, A. M. Tahir, and W. F. Faris, "Digital implementation of space vector pulse width modulation technique using 8-bit microcontroller," World Applied Sciences Journal, no. 21, pp. 21-28, 2013. [Online]. Available: https://bit.ly/2RPZF87

[25] L. Fan, Control and Dynamics in Power Systems and Microgrids. CRC Press, 2017. [Online]. Available: https://bit.ly/3yDXpBt

[26] A. Yazdani and R. Iravani, Voltage-sourced converters in power systems: modeling, control, and applications. John Wiley \& Sons, 2010. [Online]. Available: https://bit.ly/3fQU7SP 